bei welchem, passende Nebenbedingungen vorausgesetzt, die Geschwindigkeit der Entropieproduktion dem höchsten relativen Minimum entspricht. Das würde heißen, in einem Diagramm: Geschwindigkeit der Entropieproduktion gegen die in Frage kommenden Parameter - stellt der stabilste Zustand nicht einen Berggipfel dar, sondern den höchstgelegenen tiefsten Punkt aller vorhandenen Mulden. Offenbar braucht der stabile Zustand nicht stationär zu sein, sondern kann auch in Schwingungen um einen aus- gezeichneten Punkt bestehen. Dabei könnte aber das Auftreten von Schwingungen wesentlich durch sekundäre hydrodynamische Störungen bedingt sein. Aber anscheinend ist das Auftreten von Schwingungen kein rein hydrodynamisches Problem. Schwingungen an Flammen auf Brennern und in Rohrstrecken ${ }^{11}$ treten dann auf, wenn ein schwingungsfähiges System vorliegt und man sich in einem gewissen Bereich der Instabilität von Flammen befindet.

11 H. B ehrens, Z. physik. Chem. [1951], im Druck.

\title{
Über die Lichtausbeute der gasgefüllten Wolframdoppelwendellampe
}

\author{
Von F. KOREF* \\ Herrn Professor Dr. John Eggert, Zürich, zum 60. Geburtstage gewidmet
}

(Z. Naturforschg. 6 a, 405-408 [1951]; eingegangen am 28. April 1951)

\begin{abstract}
Das Prinzip der gasgefüllten Wolframglühlampe nach Langmuir verlangt die Zusammendrängung des Leuchtkörpers auf möglichst geringe Länge, eine Forderung, die sich erst nach Schaffung eines Drahtmaterials von genügender Heißformbeständ̄igkeit realisieren ließ. Der Übergang von der Einfachwendel zur Doppelwendel brachte einen großen Fortschritt in der Lichtausbeute. Durch Versuche wird gezeigt, daß dieser Fortschritt durch die Verkürzung des Leuchtkörpers und die verminderte Energieabgabe an das Füllgas nicht restlos erklärt wird. Wie aus den Versuchen ferner hervorgeht, brennen Einfachwendellampen und Doppelwendellampen gleicher Type, die auf gleiche Lebensdauer eingestellt sind, mit gleicher Leuchtkörpertemperatur. Die verschiedenen Effekte, die bei der Doppelwendel für gleiche Lebensdauer teils eine höhere, teils eine tiefere Brenntemperatur verlangen — Drahtdicke, Fangwirkung, tödlicher Gewichtsverlust - gleichen sich gerade aus. Der ungeklärt bleibende Rest der Lichtausbeutesteigerung beruht auf der geänderten Wendelform und der damit bewirkten Strahlungsverbesserung.
\end{abstract}

I. Einf achwendel und Doppelwendel

Tn der gasgefüllten Wolframlampe nach Lang-

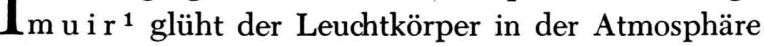
eines indifferenten Gases, z. B. Stickstoff oder Argon, durch welche seine Verdampfungsgeschwindigkeit herabgesetzt wird bzw. seine Temperatur gesteigert werden kann, während die Wattverluste durch Aufheizung des Gases dadurch verringert werden, daß der Wolframdraht in der gedrängten Form einer Wendel angeordnet wird. Nach Langmuirs Feststellungen erfolgt die Energieabgabe an das Gas nicht unmittelbar an der Drahtoberfläche, sondern im Abstand von einigen Millimetern; sie ist somit bei einer Wendel nicht der Gesamtlänge des Drahtes, sondern annähernd der Länge der Wendel proportional. Das Prinzip der Gasfüllungslampe verlangt daher die $\mathrm{Zu}$ sammendrängung des Leuchtkörpers auf möglichst

* Aarau (Schweiz), Wolframwerke M a x G loor und Glühlampenfabrik Glori a A.G.

1 J. Lang m u ir, Physic. Rev. 34, 401 [1912]. geringe Länge, eine Aufgabe, die sich erst nach Schaffung eines Drahtmaterials von genügender Heißformbeständigkeit realisieren ließ. Die in ihrer Formbeständigkeit verbesserten Drähte erlaubten es, auch bei den dünndrahtigen Wendeln für die gebräuchlichen Lampen von 40 bis 100 Watt den Wendelkern auf rund das Fünffache des Drahtdurchmessers zu steigern. Bei Bemessung des Windungsabstandes gleich etwa dem halben Drahtdurchmesser ergab sich so eine Zusammendrängung (Verhältnis von Drahtlänge zu Wendellänge) von 12 bis $14 \mathrm{zu} 1$.

Über die zahlreichen Versuche zur weiteren Verkürzung des Leuchtkörpers, die wir in der Studiengesellschaft des Osramkonzerns gemeinsam durchgeführt haben, hat mein langjähriger Mitarbeiter Moers in seiner Abhandlung „Über das Problem der Leuchtkörperzusammendrängung in gasgefüllten Glühlampen “ ausführlich berichtet ${ }^{2}$. Diese Versuche

\footnotetext{
2 K. M o e r s, Licht 8, 17 [1938].

3 DRP 422437 [1924].
} 


\begin{tabular}{|c|c|c|c|c|c|}
\hline \multirow[b]{2}{*}{ Type } & \multicolumn{2}{|c|}{$\begin{array}{c}\text { Lichtstrom } \\
\text { (Lumen) }\end{array}$} & \multicolumn{2}{|c|}{$\begin{array}{l}\text { Lichtausbeute } \\
\text { (Lumen/Watt) }\end{array}$} & \multirow{2}{*}{$\begin{array}{c}\text { Ver- } \\
\text { besse- } \\
\text { rung } \\
0 \%\end{array}$} \\
\hline & $\begin{array}{l}\text { Einfach- } \\
\text { wendel }\end{array}$ & $\begin{array}{l}\text { Doppel- } \\
\text { wendel }\end{array}$ & $\begin{array}{c}\text { Einfach- } \\
\text { wendel }\end{array}$ & $\begin{array}{l}\text { Doppel- } \\
\text { wendel }\end{array}$ & \\
\hline 2 & & & 8 & 10 & 2 \\
\hline $220 \mathrm{~V} \quad 60$ & 5 & 6 & 9 & 11 & 1 \\
\hline $220 \mathrm{~V} 75 \mathrm{~J}$ & 80 & 905 & 10 & 12,1 & 13 \\
\hline $220 \mathrm{~V} 100$ & 1180 & 1290 & 11,8 & 12,9 & 9,5 \\
\hline
\end{tabular}

Tab. 1. Steigerung der Lichtausbeute beim Übergang von der Einfachwendel zur Doppelwendel.

führten zur Schaffung der Doppelwendellampe ${ }^{3}$, bei welcher die Zusammendrängung auf etwa $30-32$ zu 1 gesteigert wird. Dabei ist allerdings zu berücksichtigen, daß beim Übergang von der Einfach- zur Doppelwendel der Draht nicht nur dicker (rund 7\% Durchmesser bei der Type 220 Volt 40 Watt), sondern auch länger (rund $12 \%$ bei dieser Type) zu bemessen ist. Dies wird nötig, weil bei zunehmender Verkürzung des Leuchtkörpers weniger Energie an das Gas abgegeben wird und bei gleichzuhaltender Wattaufnahme dafür mehr Watt ausgestrahlt werden müssen, was eine Vergrößerung der strahlenden Oberfläche erfordert, wenn die Leuchtkörpertemperatur nicht wesentlich erhöht werden soll. - Man wählt den Windungsabstand primär und sekundär größer als bei der Einfachwendel, den Kern dagegen kleiner, nämlich nur etwa gleich dem Doppelten des Draht- bzw. Primärgewickel-Durchmessers.

Die Entwicklung der Doppelwendellampe bis zur Betriebsreife erforderte eine Reihe von Jahren. Ihre Einführung erfolgte gegen die Mitte der dreißiger Jahre. Die beträchtliche Verbesserung ihrer Wirtschaftlichkeit im Vergleich zur Einfachwendellampe bei Einstellung auf gleiche Lebensdauer zeigt Tab. ${ }^{4}$.

Diese Qualitätsdifferenzen wurden in langjährigen statistischen Beobachtungen und Aufzeichnungen erhärtet.

\section{Experimentelle Untersuchungen}

über die Qualitätsdifferenz zwischen Einfach- und Doppelwendellampen

Es war nun von Interesse, zu prüfen, wie diese empirisch-statistische Qualitätsdifferenzen in der Energiebilanz beider Lampenarten zum Ausdruck kommen unter besonderer Berücksichtigung der Wattabgabe an das Gas im Sinn des Langmuirschen Prinzips. Wir wollen diese Frage an Hand einer Versuchsreihe, die bereits vor Jahren mit der Type $220 \mathrm{~V}$ 40 Watt durchgeführt wurde, näher behandeln.

4 R. S e wig, Hdb. Lichttechn. 1938, 1014.
Es wurcien je 10 Lampen mit Einfachwendeln und Doppelwendeln zum Versuch herangezogen, deren Lichtausbeute die gleiche Lebensdauer für beide Sorten erwarten ließ. Bei beiden wurde die Wattabgabe an das Gas (etwa 90\% Argon von $650 \mathrm{~mm} \mathrm{Hg}$ Druck) ermittelt, indem bei gleicher Leuchtkörpertemperatur mit und ohne Gasfüllung Spannung, Stromstärke und Lichtstrom gemessen wurden. Die Einstellung auf gleiche Temperatur erfolgte mit Hilfe des Quotienten aus Heiß- und Kaltwiderstand. Der Heißwiderstand $\left(R_{\mathrm{H}}\right)$ ergab sich aus Spannung und Stromstärke. Der Widerstand bei Zimmertemperatur $\left(R_{20}\right)$ wurde an den gealterten Lampen (Leuchtkörper gut rekristallisiert) unter genauer Innehaltung von $20^{\circ} \mathrm{C}$ an einer Wheatstoneschen Brücke gemessen. Die Sicherheit dieser Temperatureinstellung ist auf etwa $10^{\circ}$, entsprechend etwa $2 \%$ Lichtausbeute, zu schätzen. Die Temperatur der gasgefüllten Einfachwendellampen wurde als Bezugswert gewählt, die Doppelwendellampen durch leichte Volt-Änderung auf den gleichen Quotienten $R_{\mathrm{H}} / R_{20}=14,0\left(2580^{\circ} \mathrm{K}\right)$ eingestellt. Zur Ermittelung der effektiven Wendellänge wurden einige gemessene Lampen geöffnet und die Wendeln hart an den Elektroden abgeschnitten. Die Mittelwerte der Messungen sind in Tab. 2 zusammengestellt.

\section{Diskussion der Ergebnisse}

Aus Spalte 9 der Tab. 2 kann man das wichtige Ergebnis entnehmen, daß die auf gleiche Temperatur eingestellten gasgefüllten Einfach- und Doppelwendellampen in großer Annäherung die statistisch für gleiche Lebensdauer ermittelte Differenz der Lichtausbeute zeigen, nämlich mit 8,4 bzw. $10,2 \mathrm{~lm} / \mathrm{W}: 21,5 \%$ statt $21 \%$. Die Erhöhung der Lichtausbeute bei der Doppelwendel beruht also nicht etwa auf einer höheren Brenntemperatur.

Diese Tatsache war keineswegs vorauszusehen. Im Gegenteil, es war damit zu rechnen, daß die Belastbarkeit des Leuchtkörpers, d. h. seine Brenntemperatur für gleichgehaltene Lebensdauer, durch verschiedene Momente geändert werden sollte. So haben wir oben gesehen, daß bei der Doppelwendel der Drahtdurchmesser rund $7 \%$ größer ist, was die Belastbarkeit entsprechend etwa $4 \% \mathrm{~lm} / \mathrm{W}$ steigern sollte. Auch ist es durchaus wahrscheinlich, daß mit der Form der Doppelwendel eine größere Fangwirkung für die verdampften Wolframatome und somit eine geringere Verdampfungsgeschwindigkeit des Leuchtkörpers verbunden ist, was gleichfalls die Belastbarkeit erhöhen sollte. - Auf der anderen Seite gibt es aber auch Momente, die sich im ungünstigen Sinne auf die Belastbarkeit der Doppelwendel auswirken, vornehmlich ihr im Vergleich zur Einfachwendel veränderter „tödlicher Gewichtsverlust". Hierunter versteht man denjenigen Gewichtsanteil des Leuchtkörpers, der bis 


\begin{tabular}{|c|c|c|c|c|c|c|c|c|c|c|c|}
\hline 1 & 2 & 3 & 4 & 5 & 6 & 7 & 8 & 9 & 10 & 11 & 12 \\
\hline Type 220 Volt 40 Watt & $\begin{array}{c}\text { Wendel- } \\
\text { länge und } \\
\text { Dicke } \\
{[\mathrm{mm}]}\end{array}$ & $\begin{array}{l}R_{20} \\
{[\Omega]}\end{array}$ & Volt & Amp. & $\begin{array}{l}R_{\mathrm{H}} \\
{[\Omega]}\end{array}$ & $\frac{R_{\mathrm{H}}}{R_{20}}$ & 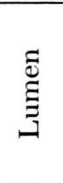 & $\operatorname{lm} / \mathrm{W}$ & Watt & 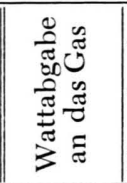 & 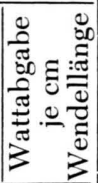 \\
\hline $\begin{array}{l}\text { a) Einfachwendel gasgefüllt } \\
\text { b) Einfachwendel entlüftet }\end{array}$ & $\begin{array}{c}53 \\
0,15\end{array}$ & 87,0 & $\begin{array}{l}220 \\
182\end{array}$ & $\begin{array}{l}0,1805 \\
0,1495\end{array}$ & $\begin{array}{l}1219 \\
1219\end{array}$ & $\begin{array}{l}14,0 \\
14,0\end{array}$ & $\begin{array}{l}333 \\
325\end{array}$ & $\begin{array}{r}8,4 \\
11,9\end{array}$ & $\begin{array}{l}39,7 \\
27,2\end{array}$ & $\left(\begin{array}{c}12,5 \\
(31,5 \%)\end{array}\right.$ & 2,4 \\
\hline $\begin{array}{l}\text { c) Doppelwendel gasgefüllt } \\
\text { d) Doppelwendel entlüftet }\end{array}$ & $\begin{array}{c}23+2,7^{*} \\
0,40\end{array}$ & 85,5 & $\begin{array}{l}218 \\
197\end{array}$ & $\begin{array}{l}0,1828 \\
0,1643\end{array}$ & $\begin{array}{l}1195 \\
1195\end{array}$ & $\begin{array}{l}14,0 \\
14,0\end{array}$ & $\begin{array}{l}408 \\
400\end{array}$ & $\begin{array}{l}10,2 \\
12,4\end{array}$ & $\begin{array}{l}39,9 \\
32,3\end{array}$ & $\left(\begin{array}{c}7,5 \\
(19,0 \%)\end{array}\right.$ & 3,2 \\
\hline
\end{tabular}

* Länge der geraden Enden aus Primärwendel

Tab. 2. Einfachwendel- und Doppelwendellampe 220 Volt 40 Watt bei Einstellung auf gleiche Leuchtkörpertemperatur.

zu dessen Durchbrennen verdampft. Er stellt eine statistische Größe dar, ebenso wie die mittlere Lebensdauer der Lampen, und wird von der Leuchtkörperanordnung - geradfädig, Einfachwendel oder Doppelwendel - wie auch von anderen Faktoren beeinflußt. Je größer der tödliche Gewichtsverlust, desto länger lebt die Lampe, bzw. um so stärker kann sie belastet werden für unveränderte Lebensdauer. Nach vorliegenden Mitteilungen ${ }^{5}$ muß damit gerechnet werden, daß der tödliche Gewichtsverlust der Doppelwendel kleiner ist als der der Einfachwendel, was ihre Brenntemperatur für gleiche Lebensdauer herabsetzt. - Unsere Messungen beweisen, daß die Faktoren, die eine Erhöhung bzw. Erniedrigung der Brenntemperatur für gleichgehaltene mittlere Lebensdauer verlangen, sich ungefähr die Waage halten und infolgedessen Einfachwendel und Doppelwendel bei gleicher Brenntemperatur die gleiche mittlere Lebensdauer haben.

Was nun die Wattabgabe an das Gas betrifft, so ergibt sie sich bei der Einfachwendel zu 12,5 Watt (31,5\% der Gesamtwatt), bei der Doppelwendel zu 7,5 Watt (19,0\% der Gesamtwatt). Je cm Wendellänge finden wir bei der Einfachwendel, deren Durchmesser $0,15 \mathrm{~mm}$ beträgt, $2,4 \mathrm{Watt}$ und bei der Doppelwendel mit einem Durchmesser von $0,40 \mathrm{~mm} \mathrm{3,2} \mathrm{Watt.}$ $\mathrm{Ge}$ is s ${ }^{6}$ hat aus den Langmuirschen Formeln für eine Gaszusammensetzung und einen Gasdruck, die von denjenigen unserer Versuchslampen nur wenig abweichen, und eine Leuchtkörpertemperatur $2700^{\circ} \mathrm{K}$

5 Vgl. R. S c h mid t, Licht 14, 19 [1944].

6 W. G e is s, Philips' Techn. Rdsch. 6, 338 [1941].
- $120^{\circ}$ höher als bei uns - folgende Werte berechnet: $2,59 \mathrm{~W} / \mathrm{cm}$ für $\varnothing 0,15 \mathrm{~mm}$ und $3,38 \mathrm{~W} / \mathrm{cm}$ für $\varnothing 0,40 \mathrm{~mm}$. Unsere Versuchsergebnisse stehen also in bestem Einklang mit der Theorie und überdies auch mit den Messungen von $\mathrm{M}$ o e r s ${ }^{2}$.

Die Energiebilanz stellt sich danach so dar, daß bei der Einfachwendel $31,5 \%$ der aufgenommenen Watt an das Füllgas abgegeben und $68,5 \%$ ausgestrahlt werden, bei der Doppelwendel dagegen entsprechend $19 \%$ und $81 \%$. Die Steigerung der Ausstrahlung von 68,5 auf $81 \%$ der aufgenommenen Energie bedeutet eine Erhöhung der Lichtausbeute um $18 \%$. Das ist aber merklich weniger als die empirisch festgestellte Verbesserung von $21 \%$ bzw. die Differenz von $21,5 \%$ bei unseren Versuchen. Die höhere Wirtschaftlichkeit der Doppelwendellampe ist also durch die Wattersparnis im Sinne von Langmuir nicht restlos belegt. Die Doppelwendel ist besser, als man rechnungsmäßig erwarten konnte. - In der Tat zeigt der Vergleich von Zeile b) und d) der Tab. 2 in Spalte 9 für die auf gleiche Temperatur eingestellten Leuchtkörper im Vakuum für die Doppelwendel mit $12,4 \mathrm{~lm} / \mathrm{W}$ eine um $4 \%$ höhere Lichtausbeute als die Einfachwendel mit $11,9 \mathrm{~lm} / \mathrm{W}$ statt der bei gleicher Temperatur im Vakuum zu erwartenden Gleichheit derselben.

IV. Deutung des nach dem Langmuirschen Prinzip nicht geklärten Teils der Qualitätsdifferenz

Die Erklärung ist in den geänderten Strahlungsbedingungen zu suchen. Während ein glühender ge- 
rader Wolframdraht die für Wolfram typische selektive Strahlung aussendet, ist die Strahlung einer Wolframwendel gemischt, die Außenseite der Wendel strahlt selektiv, die aus dem Inneren kommende Strahlung ist mehr oder weniger geschwärzt, $\mathrm{d}$. h. lichtökonomisch ungünstiger. Das Mischungsverhältnis der selektiven und schwarzen Strahlung hängt von dem Windungsabstand der Wendel ab. Dẻmgemäß ist bei gleicher Brenntemperatur die Lichtausbeute des geraden Drahtes größer als die der Wendel, und der Unterschied beträgt bis zu etwa $8 \%^{7}$. Am ungünstigsten verhält sich eine Wendel, wenn der Windungsabstand etwa die Hälfte des Drahtdurchmessers beträgt. Mit enger werdendem Abstand nimmt der Anteil der schwärzeren Innenstrahlung ab, mit größe-

7 Vgl. E. Lax u. M. Pirani, Hdb. Physik von Geiger u. Scheel XIX, 434 [1928], ferner G. R. F o n d a u. A. A. V e rn o n, J. opt. Soc. America 22, 223 [1932]. rem Abstand kann auch die Innenseite der Wendel zunehmend frei strahlen. Wird der Abstand größer als der Drahtdurchmesser, so nähert sich das Verhalten der Wendel dem des geraden Drahtes.

Bei den üblichen Einfachwendeln beträgt, wie oben erwähnt, der Abstand rund einen halben Drahtdurchmesser, die Strahlungsverhältnisse sind demgemäß am ungünstigsten. Bei der Primärwicklung der Doppelwendel wird der Abstand von vorneherein schon nahezu gleich dem Drahtdurchmesser gewählt. Durch das zweite Wickeln um einen relativ engen Kern wird die Primärwendel noch stark aufgespreizt, so daß der Wendelabstand das $1^{1} / 2^{-}$-fache des Drahtdurchmessers und mehr beträgt. Die dadurch bewirkte Verbesserung der Strahlungsökonomie kann wohl auf etwa 4\% der Lichtausbeute geschätzt werden, wodurch der nach dem Langmuirschen Prinzip nicht geklärte Teil der Qualitätssteigerung seine Deutung findet.

\title{
BE RICHTE
}

\section{Photographic Sensitivity and Chemical Sensitisation of Emulsions*}

\author{
By W. F. Berg \\ Research Laboratory, Kodak Limited, Harrow, Middlesex (England) \\ (Z. Naturforschg. 6 a, 408-411 [1951]; eingegangen am 1. Juni 1951) \\ To Professor John Eggert for his $60^{\text {th }}$ birthday
}

\begin{abstract}
A $s$ a result of the war, a considerable amount of material has been published which would normally be hidden behind the silver curtain 1 that guards the emulsion makers' secrets; this material was discussed recently at a conference held at B ris to ${ }^{2}$ and it may be useful to attempt to give a coherent picture of our knowledge in this field.

Unfortunately, as mostly in photographic theory, it is necessary to adopt deductive reasoning, the process being too complicated to allow the fundamental mechanism to be derived from experimental experience. This inevitably lends our picture an indirect and unsatisfactory atmosphere, and few points are capable of direct and unequivocal proof. It might be safer to label any of the mechanisms discussed as working hypotheses rather than as fully fledged theories.

The Gurney-M ot t theory ${ }^{3}$ has found almost universal acceptance in describing the mechanism of latentimage formation in a dry emulsion layer; it quite

* Based on the Renwick Memorial Lecture of the Royal Photographic Society, held in Birmingham, England, on 18. 4. 1951.

1 With apologies to Dr. H. B a in es for this plagiarism.

2 'Fundamental Mechanisms of Photographic Sensitivity' Butterworth, London 1951.
\end{abstract}

deliberately avoids the discussion of the preparation of the sensitive emulsion. It is well known that this theory visualises the latent image as a small speck of silver which is produced during exposure by the separate movement of photo-electrons and silver ions, which condense on a nucleus, such as for example a speck of silver or silver-sulphide, which acts by trapping the electron. It is interesting to speculate upon the fact that E g g e r t ${ }^{4}$ suggested very much the same mechanism in 1926, but this was not taken seriously at the time, apparently even by its originator, and was soon forgotten. It seems that the time was simply not ripe for this advance in thought.

$$
\begin{gathered}
\text { The definition of photographic } \\
\text { sensitivity }
\end{gathered}
$$

We are here interested in the number of quanta which an emulsion grain must absorb to become developable under given conditions of exposure and processing; sensitivity in our sense is defined in terms of this number.

3 Gurney and Mott, Proc. Roy. Soc. [London], Ser. A 164, 151 [1938].

4 Eg gert, Z. Elektrochem. angew. physik. Chem. 32, 491 [1926]. 